\title{
Fit 4 surgery, a bespoke app with biofeedback delivers rehabilitation at home before and after elective lung resection
}

\author{
Salma Bibi Kadiri', Amy Pamela Kerr', Nicola Katy Oswald ${ }^{2}$, Alina-Maria Budacan ${ }^{1}$, Sarah Flanagan³, \\ Christopher Golby ${ }^{4}$, Stuart Lightfoot ${ }^{1}$ and Babu Naidu ${ }^{1,2^{*}}$
}

\begin{abstract}
Background: Pulmonary rehabilitation programme for lung surgery patients can reduce the risk of post-operative complications but compliance to programmes can be limited by access to health care. We developed a homebased rehabilitation app and tested its feasibility in patients undergoing lung resection surgery.

Methods: A cohort study was conducted over 18 months at a regional thoracic unit. The Fit 4 Surgery app included ten exercises. Patients were instructed to exercise for at least three minutes for each exercise. Data was transmitted back to the researchers remotely. Data was also collected from a contemporaneous group of surgery patients who attended local outpatient-based Chronic Obstructive Pulmonary Disease rehabilitation classes. Quality of Life and outcomes data in the app group were collected. Patients were also interviewed about their experience of the app.
\end{abstract}

Results: App patients had a shorter wait before surgery compared to patients attending rehabilitation classes (24 vs 45 days) but managed four times as many sessions (2 vs 9), improving incremental shuttle walk test distance by $99 \pm 83(p<0.05)$ metres before surgery. Five themes were gathered from the interviews.

Conclusion: An app based programme of rehabilitation can be delivered in a timely fashion to lung surgery patients with demonstrable physiological benefits; this will need to be confirmed in further clinical trials.

Clinical trial registration number: ISRCTN00061628. Registered 27 May 2011.

Keywords: Pulmonary rehabilitation, Lung Cancer, Exercise, Thoracic surgery, Quality of life, Intervention, Technology

\section{Background}

Up to $15 \%$ of patients develop post- operative complications after lung surgery which can result in death, admission into an intensive care unit, prolonged hospital stay and readmission to hospital after discharge $[1,2]$. The evidence on pre- and post-operative rehabilitation in lung resection surgery is poor, mainly due to the heterogeneity of patient population, interventions and outcomes

\footnotetext{
* Correspondence: b.naidu@bham.ac.uk

'Department of Thoracic Surgery Research, Heartlands Hospital, Bordesley Green East, Birmingham B9 5SS, UK

${ }^{2}$ Institute of Inflammation and Ageing, College of Medical and Dental Sciences, Centre for Translational Inflammation Research, University of Birmingham Laboratories, Queen Elizabeth Hospital Birmingham, Edgbaston, Birmingham B15 2TT, UK

Full list of author information is available at the end of the article
}

[3]. Recently published Enhanced Recovery After Surgery guidelines recommend the use of prehabilitation for patients with borderline lung function or exercise capacity [4]. Meta-analysis of studies of pulmonary rehabilitation and/or exercise classes for lung surgery patients demonstrate improvement in exercise capacity, quality of life and a reduction in post-operative complications $[5,6]$. However, access to rehabilitation services can be hampered because of limitations in local health care resources and patient reluctance to attend multiple classes. Rehabilitation services can find it difficult to deal with the fluctuation in demand from flow of lung cancer surgery patients so can struggle to provide a consistent service [7].

Therefore, there is a need for a service that can be delivered immediately at the convenience and in the 
control of the patient. Mobile device use in the healthcare sector to access systems has rapidly evolved with an explosion in software applications (apps). Apps have been shown to support education for self-management and provide extensive feedback to users to facilitate behaviour change $[8,9]$. Physical activity interventions incorporating technology have been used with varying degrees of success in the medical disease setting but not in the surgical arena [5]. Knowing that pulmonary rehabilitation consisting of exercise classes is beneficial to lung surgery patients but is resource limited and that apps can promote physical exercise in other disease settings, we sought to develop a bespoke pulmonary rehabilitation app and test its feasibility and acceptability to patients undergoing lung resection surgery.

\section{Methods}

An ethically approved cohort study (Research Ethics Committee reference 10/H1208/41) was conducted over 18 months at a regional thoracic unit. Inclusion criteria were broad; any patient deemed eligible for curative lung cancer surgery based on British Thoracic Society guidelines by the multidisciplinary teams referring to a regional thoracic surgery unit.

Surgery was never delayed for the purposes of the study, thus time spent using the app varied. Patients recommenced the programme 2 weeks after discharge. Data on adherence to local COPD pulmonary rehabilitation classes was also collected from a contemporaneous group of patients, who had agreed to attend these sessions twice a week up to the day of their surgery and for 6 weeks following surgery as described previously [7]. The only comparison made between the two groups pertained to the process measures (ie: number of sessions pre and post surgery).

\section{Intervention}

The 'Fit 4 Surgery' app consisted of ten exercises, both upper and lower limb, aerobic and strength, based on the lung cancer 'Rehabilitation for Operated lung Cancer' surgery (ROC) programme and available on the patient website (http://www.thoracicsurgery.co.uk) [5].

The app was developed for an Ipad mini 2 cellular (Apple Inc. California, USA), configured with a blue tooth enabled pulse oximeter (Creative PC-68B, Shenzhen Creative Industry Co. Ltd., China) and a Subscriber Identity Module (SIM) card to enable wireless feedback of data to the researchers. The app collected baseline measurements of oxygen saturation and heart rate for safety. It provided the patient with a target heart rate (> $60 \%$ of maximum heart rate) based on their age to be achieved during each exercise and patients were given all equipment.
Each exercise was demonstrated to the patient through a series of video clips on the app which they could follow whilst the pulse oximeter collected heart rate and oxygen saturation levels continuously (Fig. 1).

The patients were instructed to exercise for at least three minutes per exercise. After each exercise, a summary screen provided feedback to patients on how effective they were at completing the exercise. This included: duration of exercise, average oxygen saturation and whether they reached their target heart rate. At the end of each exercise patients could rate their effort by completing a Borg scale breathlessness questionnaire on the app [10].

At the end of each session, a summary of all exercises completed in that session was displayed. Patients completed a series of questions rating their overall exercise session and could leave any further comments through an audio recording captured through the ipad. Anonymous data from the app was transferred wirelessly on to a cloud based server compliant with National Health Service (NHS) information governance guidance. Data from this server could be accessed by the research team. Videos from the ROC education programme were incorporated into the app, to inform patients about the surgery, importance of exercise; patient pathway [7]. Patients in the contemporaneous rehabilitation class group also received this information by a written leaflet and/or reference to the website, and/or provision of a DVD.

\section{Development}

The exercise programme was refined in an iterative process by a stakeholder group consisting of thoracic surgeons, physiotherapists, patient representatives, thoracic surgery nurses, and app developers. This group met at regular time intervals during the project to develop and refine the app and its operations. The app was tested in a hospital pulmonary rehabilitation class with five lung surgery patients and feedback gathered on ease of use and 'bugs' in the system. Thus an iterative process of building functionality was followed and feedback incorporated into the second stage of development to refine the performance of the app. The app was created using the Swift 3 coding language and was compatible with the iOS 11 operating. App data was transferred to a RESTful (Representational State Transfer) web service, which allows data to be stored in the cloud, and then accessed through a standard web browser.

\section{App training}

Patients were registered on the app and their baseline heart rate was measured. Registration, in addition to baseline demographics, included a series of questions regarding co-morbidities with generated appropriate pop up messages with contraindications and advice relevant 
A

Using The App

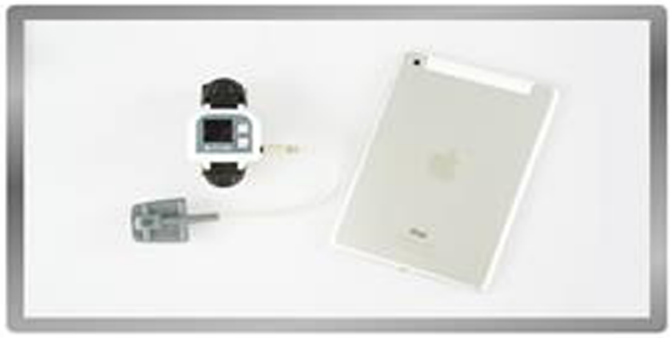

C

\section{$\equiv$}

(i)

02 Saturation

$96 \%$

Heartrate (bpm)

800

Completed

00:15

(2) (2)

B

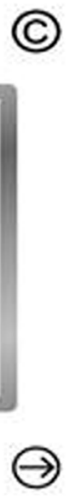

D

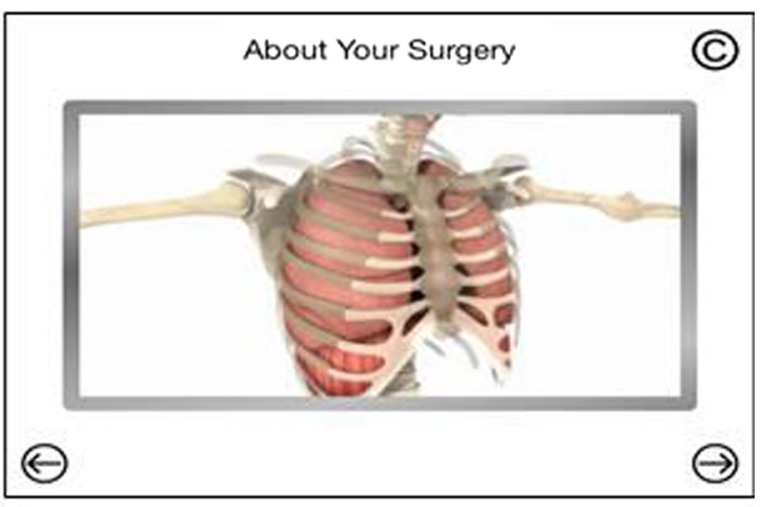

$\mathbf{F}$

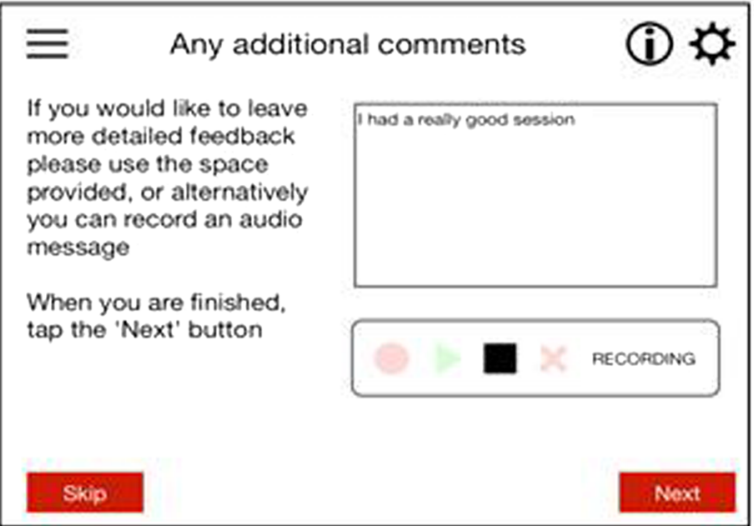

E

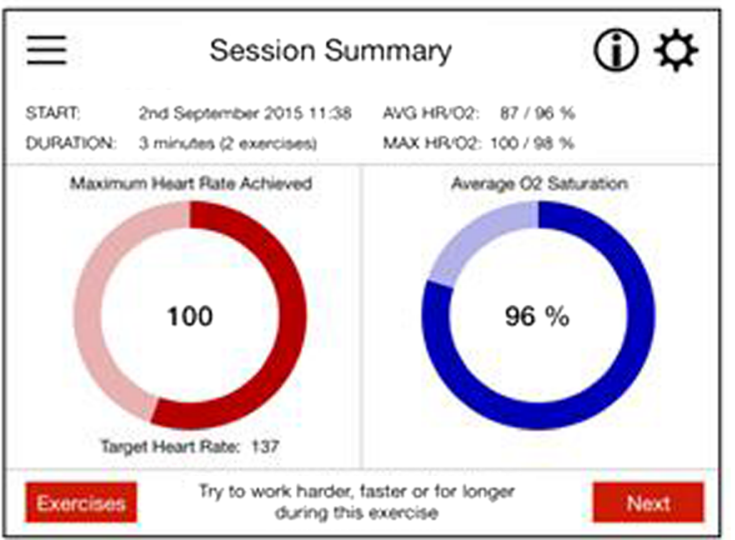

Fig. 1 (See legend on next page.) 
(See figure on previous page.)

Fig. 1 Screenshots from Fit 4 Surgery App. a training video for patients on how to use the app. $\mathbf{b}$ safety notification screen (c) one of the exercise videos which shows feedback e.g. duration of exercise, heart rate and $\mathrm{O} 2$ saturation. $\mathbf{d}$ one of the education videos that patients can view using the app. e a summary screen post exercise which shows if target heartrate was achieved, average $\mathrm{O} 2$ saturation and motivation feedback. f Additional comments box screenshot

to specific conditions (Fig. 1). Patients were taught how to use the app by the researcher. This was delivered from a scripted training document to ensure consistency. In addition to the one-to-one consultation, patients were given an instruction booklet with details on how to use the app and troubleshooting issues (Additional file 1). Patients were called once a week whilst using the app by the research team to assess if they had any technical problems.

\section{Contemporaneous 'rehabilitation class' group}

Patients were enrolled into local COPD rehabilitation classes in a pragmatic fashion; these could take place in the hospital or community based setting and in a group or individual class depending on the services offered locally [5]. All classes followed the national guidance on COPD rehabilitation; they were scheduled twice a week, lasted $90 \mathrm{~min}$ and included both strength and aerobic exercises for both the upper and lower body. These exercises were similar to those used in the app. Data on process measures from these classes were retrospectively collated.

\section{Assessments}

Demographic data was collected for the app group as follows: age, COPD, ischaemic heart disease, body mass index (BMI), smoking status, surgery and analgesia type, modified Medical Research Council (mMRC) dyspnoea scale, Eastern Cooperative Oncology Group (ECOG) performance status, spirometry and pathology.

Delivery of the exercise programme, testing usability and evaluation of the improvement was established in a mixed-method approach and performed along the following lines:

\section{Process measures}

Measures including time taken from identification of patient to first rehabilitation day and number of days of rehabilitation before and after surgery were noted.

\section{Physiological parameters}

App patients completed an incremental shuttle walk test (ISWT) and spirometry before surgery, pre-and postrehabilitation. Frequency, type and duration (minutes) of exercises completed using the app in each session were recorded.

\section{Patient experience of app and effect on QOL effects / usability}

Semi-structured telephone interviews were undertaken with 13 patients by an independent researcher to avoid bias and increase validity of the information collected. Interviews were undertaken 2 weeks after patients finished using the app. All interviews were digitally recorded and transcribed. The transcripts were analysed using content analysis. The following areas of interest were explored with participants: motivation for using the app: effectiveness of staff communication and written communication about how to use the app, usability of the app, impact of the app upon perceived (and actual) levels of fitness, any specific problems encountered using the app and any factors that influenced their use of the app, what aspects of the app were particularly useful and recommendations for changes to improve the app.

Patient quality of life was measured using European Organisation for Research and Treatment of Cancer Quality of Life Questionnaire 30 (EORTC-QLQ30) pre and post rehabilitation pre-surgery and, 6 weeks' and 5 months' post-surgery.

\section{Outcome measures}

Inpatient length of stay, rate of postoperative pulmonary complication (PPC), ITU admissions, hospital length of stay and readmission to hospital within 30 days of surgery were recorded in the app group.

\section{Statistical analysis}

Data presented is summarised using appropriate parametric and non-parametric methods. The only intergroup comparison was made in relation to process measures.

\section{Results}

Characteristics of the app group are shown in Table 1.

Process measures (Tables 2 and 3)

Patients in the app group waited a median of 6 (range 13-33) days to receive the app and completed a median of 9 (range 1-37) sessions of exercises on the app before surgery and a median of 4 (range 1-7) sessions per week. Patients in the class group waited a median of 5 (range 0-23) days to be seen in a rehabilitation class and attended 4 classes (range 1-15). However, 32\% [11] of patients did not use the app post-surgery. For the 
Table 1 Demographics

\begin{tabular}{|c|c|}
\hline & App group $(n=31)$ \\
\hline Age, mean (SD) & $64(12)$ \\
\hline BMI, mean (SD) & $25.7(9.6)$ \\
\hline$\%$ predicted FEV1, mean (SD) & $74.2(34)$ \\
\hline Measured FVC, mean (SD) & $3.40(1.14)$ \\
\hline$\%$ predicted DLCO, & $68.2 \%$ \\
\hline \multicolumn{2}{|l|}{ Pathology } \\
\hline NSCLC & $54.8 \%(17)$ \\
\hline Other lung Ca & $22.6 \%(7)$ \\
\hline Metastatic & $3.2 \%(1)$ \\
\hline Benign & $19.4 \%(6)$ \\
\hline \multicolumn{2}{|l|}{ Self-reported pre- op activity level \% (N) } \\
\hline Able to walk $<400 \mathrm{~m}$ & $26 \%(8)$ \\
\hline Able to walk at least $400 \mathrm{~m}$ & $19 \%(6)$ \\
\hline Able to walk at least $2 \mathrm{~km}$ & $6 \%(2)$ \\
\hline Able to walk $>2$ Kilometres & $48 \%(15)$ \\
\hline \multicolumn{2}{|l|}{ Smoking \% (N) } \\
\hline Never & $19.4 \%(6)$ \\
\hline Ex $>6$ weeks & $41.9 \%(13)$ \\
\hline Ex $<6$ weeks & $12.9 \%(4)$ \\
\hline Current & $25.8 \%(8)$ \\
\hline Ischaemic heart disease \% (N) & $6.5 \%(2)$ \\
\hline COPD \% (N) & $27.6 \%(9)$ \\
\hline mMRC score, median (IQR) & $1(0-1)$ \\
\hline ECOG performance status, median (IQR) & $0(0-1)$ \\
\hline
\end{tabular}

BMI Body Mass Index, FEV1 Forced expiratory volume, FVC Forced lung capacity, NSCLC Non-small cell lung cancer, DLCO Diffusing capacity of the lung for carbon monoxide, COPD Chronic Obstructive Pulmonary disease, ECOG the Eastern cooperative oncology group

patients who had attended rehabilitation classes' presurgery, $79 \%$ did not attend rehabilitation classes postsurgery. Exercise measures are displayed in Table 2. In summary, app patients had a shorter time before surgery ( 24 vs 45 days) but managed to complete more than 4 times as many exercise sessions (2 vs 9 ) and completed more sessions after surgery ( 2 vs 0$)$ than those in the class group.

\section{Physiological parameters}

Baseline median ISWT in the app group was $367 \mathrm{~m}$ (IQR105-480) prior to rehabilitation and improved to
Table 3 Exercise parameters in app group

\begin{tabular}{lll}
\hline App- exercise parameters & Median (\%) & Range \\
\hline Number of sessions pre-surgery & 9 & $1-37$ \\
Number of sessions per week pre-surgery & 4 & $1-7$ \\
Total exercise time (mins) pre-surgery & 158 & $3-1226$ \\
Time spent in target heart rate of $\geq 60 \%(\%)$ & 32 & $3-93$ \\
Number of exercises pre -surgery & & \\
Upper body & $14.5(46.3)$ & $1-73$ \\
Lower body & $21.5(53.7)$ & $1-71$ \\
Median number of sessions post-surgery & 2 & $0-30$ \\
Median number of sessions per week post-surgery & $<1$ & $0-4$ \\
Total exercise time (mins) post-surgery & 22 & $0-888$ \\
Median Number of exercises post-surgery & & \\
Upper body & $4(47)$ & $0-90$ \\
Lower body & $5(53)$ & $0-87$ \\
\hline
\end{tabular}

$450 \mathrm{~m}$ (IQR169-680) following app rehabilitation, prior to surgery (Fig. 2). There was no relationship between magnitude of increase in the ISWT and total exercise time or time spent at target heart rate during exercise or any other exercise parameter.

\section{Patient experience of app and effect on QOL effects / usability (see Fig. 3)}

Interviews were conducted until saturation was achieved $(n=13)$. Seven females and six males were interviewed.

\section{Motivation for taking part in the study}

There were six motivations for patients' participation in the study.

\section{Ease of use/instructions to use the app}

All the patients found the verbal and written communication, straightforward. They felt that the research team fully explained how to use the app. All but 3 patients had some experience of using digital technologies to varying degrees (from using a smartphone to using smart devices for texting and using apps). One patient had experience as a web developer and was impressed with the app. Other patients with less experience also found it easy to use.

Table 2 Process measures

\begin{tabular}{|c|c|c|}
\hline & $\operatorname{App}(N=31)$ & Classes $(N=34)$ \\
\hline Days from screened to 1st rehab, median (IQR) & $6(2-13)$ & $5(0-23)$ \\
\hline Days from rehab to surgery date, median (IQR) & $24(13-33)$ & $45(27-71)$ \\
\hline Rehab sessions before surgery, median (IQR) & $9(6-13)$ & $2(1-8)$ \\
\hline Days of rehab post-surgery, median (IQR) & $2(0-7)$ & $0(0-0)$ \\
\hline
\end{tabular}




\section{Incremental Shuttle Walk test before and after rehabiitation ( pre surgery)}

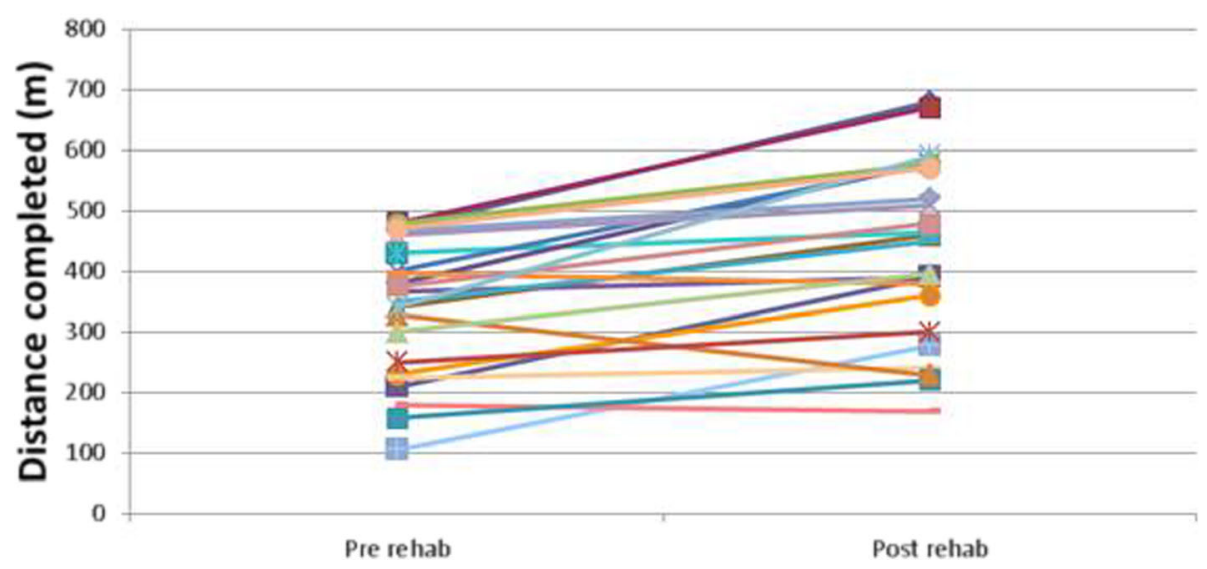

Fig. 2 Incremental shuttle walk test before and after rehabilitation in app group. Each patient is represented by one line

\section{Reported positive aspects and features of the app}

The app enabled patients to set their own pace. The simplicity of the app meant that the exercises were repeatable. Many patients found that being able to see their oxygen levels and heart rate was motivational and the variety of exercises was also welcomed. The novelty factor of using the app for exercise was appealing to some patients and even patients who had good levels of fitness prior to using the app found it beneficial.

\section{Problems and tips for improvements}

Despite the overall ease of use, patients identified some minor areas that may improve patient experience and utility of using the app and these were incorporated into the app on subsequent iterations.

\section{Perceived impact upon fitness levels}

Patients had varied levels of fitness prior to surgery but overall, almost all reported benefit from using the app. Success seemed to be contingent upon both the ease of use, personal levels of motivation and health status.

\section{Quality of life scores (Table 4)}

All of the scales and single-item measures ranged in score from 0 to 100 . A high scale score represented a higher response level; a high score in the functional scale is classed as a healthy level of functioning, a high score for a symptom item represents a greater level of symptomatology. A change in any scale of at least 10 is considered clinically relevant [10]. The changes in scores pre surgery to 5 weeks post-surgery to 5 months post op in the app group are shown in Table 4. Of note, the Global Health score at 5 months for the app significantly increased and had returned to baseline level.
Outcome measures (Table 5)

Inpatient length of stay, rate of postoperative pulmonary complication (PPC), ITU admissions, hospital length of stay and readmission to hospital within 30 days of surgery were recorded in the app group. $48.4 \%$ of patients underwent lobectomy and the same percentage benefited from a sublobar resection, either via VATS $(54.8 \%)$ or thoracotomy $(45.2 \%)$. The median length of stay was 4 days and the PPC rate was $9.7 \%$.

\section{Discussion}

The objective of this Fit 4 Surgery app study was to develop, refine and examine the feasibility of an app that could deliver a pulmonary rehabilitation/exercise programme for operated lung cancer patients, in the comfort of their homes, safely and effectively. The fact that patients in the app group managed more sessions during the pre- and post-op period compared with the rehabilitation group, demonstrates that it is feasible to deliver the intervention and is acceptable and compliant to patients. Thirty one patients used the app and a separate 34 patients from the same surgical cohort attended exercise classes in the same period. No statistical comparisons have been made between groups but process measures data from both groups is presented to highlight issues in both types of rehabilitation.

The low number of rehabilitation classes attended prior to surgery highlights the difficulties of getting patients to classes. In the app group the system is efficient and effective at delivering exercise sessions despite a shorter time period to surgery. Furthermore, if we exclude the 3 poorly compliant patients with an exercise time of less than $10 \mathrm{~min}$, the median total exercise time before surgery rises from 158 to $194 \mathrm{~min}$. The drop-out 


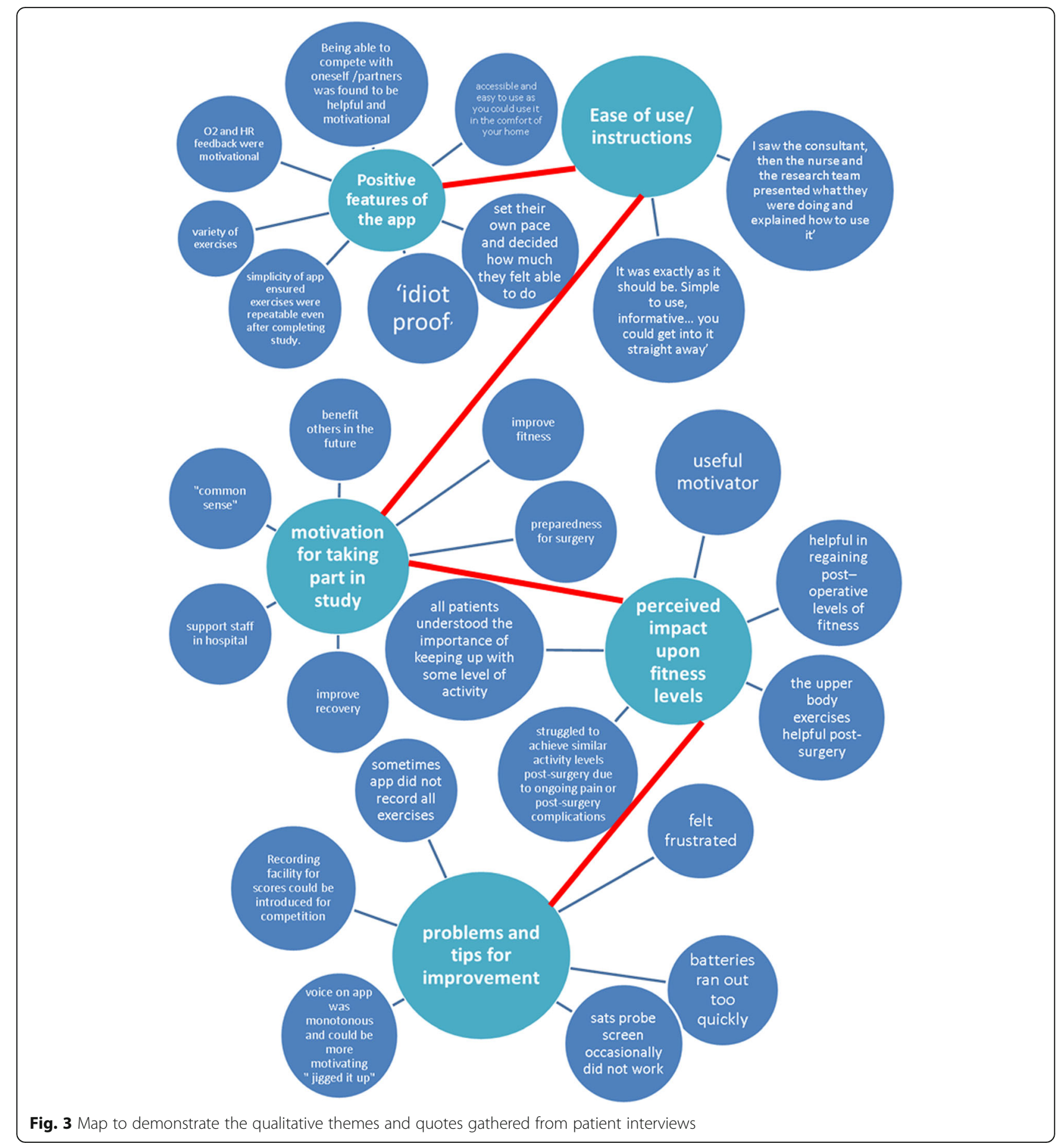

rate of patients in the app group from restarting exercise after surgery was significantly lower than compared to class rehabilitation group ( $32 \%$ vs $79 \%$ ). From the patient interviews, reasons for not continuing app-exercises after surgery were: pain, lack of motivation and generally feeling unwell. Further work is required in adapting the app to deal with the changing needs and motivation to exercise after surgery. To promote optimal and sustained behaviour change in physical activity, it is essential that interventions target recognised determinants and are theoretically grounded [11]. Behavioural change techniques (e.g., goal setting) underpinned by a sound theoretical basis need to be embedded within the app to improve efficacy [11].

Previous studies have shown a plethora of exercise interventions ranging from 12 to 20 classes (over 4 to 6 weeks) of combination strength and endurance exercise training and other adjuncts. Patients using the app in 
Table 4 EORTC QLQ C30 scores in the app group

\begin{tabular}{lll}
\hline Domain & Change in scores pre surgery to 5 weeks post-surgery & Change in scores post op 5 weeks to 5 months \\
\hline Physical & $19.0(17.8)$ & $-4.73(27.7)$ \\
Role & $19.3(30.2)$ & $-4.98(41.5)$ \\
Fatigue & $-23.4(26.4)$ & $3.88(35.2)$ \\
Pain & $-18.4(33.2)$ & $15.7(32.6)$ \\
Dyspnoea & $-26.8(28.0)$ & $1.7(42.7)$ \\
Global Health status & $19.6(19.1)$ & $-10.0(29.8)$ \\
\hline
\end{tabular}

Mean (standard deviation) change in QOL scores from pre rehabilitation to 5 months post-surgery

our study received on average 9 sessions which is less than the above studies but ISWT distances post rehabilitation before surgery increased by $99 \mathrm{~m}$. This is above the minimally important clinically difference and is on par with other studies [12-14].

Attributing increases in ISWT results purely to exercise time captured by the app may be misleading as patients may have made broader lifestyle changes as other have described in other physical activity apps [15]. Thus, in subsequent studies it will be important to measure overall physical activity changes.

Physical activity interventions incorporating technology are actively being used in patients, mostly commonly with COPD, asthma and cardiovascular diseases [16-18]. Apps can successfully deliver patient education, disease self-management, and assist in the remote monitoring of patients $[19,20]$. Low cost 'off the shelf' wearable technology (sensors) can track biometric data such as heart

Table 5 Outcome measures

\begin{tabular}{ll}
\hline & App (N=31) \\
\hline Surgery \% (N) & \\
Lobectomy & $48.4 \%(15)$ \\
Sublobar & $48.4 \%(15)$ \\
Pneumonectomy & $3.2 \%(1)$ \\
Analgesia \% (N) & \\
Epidural & $41.9 \%(13)$ \\
$\quad$ Morphine infusion & $6.4 \%(2)$ \\
$\quad$ Paravertebral & $51.6 \%(16)$ \\
Surgical technique \% (N) & \\
$\quad$ Thoracotomy & $45.2 \%(14)$ \\
$\quad$ VATS & $54.8 \%(17)$ \\
Length of stay, median (IQR) & $4(3-7)$ \\
PPC rate \% (N) & $9.7 \%(3)$ \\
Unplanned ITU rate \% (N) & $6.4 \%(2)$ \\
Unplanned ITU LOS in days' median (IQR) & $1.5(1-2)$ \\
30 day hospital readmission \% (N) & $9.7 \%(3)$ \\
$30-$-day mortality \% (N) & $0 \%(0)$ \\
\hline
\end{tabular}

PPC Postoperative Pulmonary complication, ITU Intensive therapy unit, LOS length of stay, VATS video-assisted thoracosopic surgery rate or steps and can be used in conjunction with apps to enable users to monitor their physical activity levels and progress. However, there is a shortfall of validated medical apps that present such sensor-based information to the user.

In one study, pulmonary rehabilitation was delivered using the LungFIT app on a smartphone. Heart rate and oxygen saturations were also measured using an integrated pulse oximeter [21]. However, the accuracy of the LungFIT physiological measurements were unreliable as the finger probe kept falling off during exercise. For the Fit 4 surgery study, we used a sturdy finger probe with a wrist strap attachment to ensure there were minimal movements during exercise to avoid spurious results. The Lung FIT app was tested in a healthy population; COPD and cardiovascular disease were exclusion criteria. Fit 4 Surgery app was tested in patients with multiple co morbidities. Furthermore, LungFIT was tested in a younger population (average age 47 years), consisting of researchers, family and friends, thus potentially creating bias. The LungFIT app was used in a laboratory environment and measurements collated by the researchers, not automatically or remotely as we have done in our study. Fit 4 surgery app was successfully delivered, in the patient's home environment. There were no adverse or serious events, relating to using the app at home. Until now it was unclear as to whether a rehabilitation app is feasible in an older patient population in a home setting and our study shows that this is possible.

A major limitation of this feasibility study was that it was not powered to look at differences in clinical outcomes as the rehabilitation class cohort outcome measures were not comparable to the app group. Should further studies demonstrate this app makes a difference in clinical outcomes, we plan to make it freely available. The cost of implementation when this intervention is at the stage of being locally tailored and scaled up relates mainly to the extra amount of time a lung cancer nurse or physiotherapist would have to spend with the patient. From experience with our pilot study, we estimate that a total of $60 \mathrm{~min}$ allied health care professional contact time is required during the whole programme per patient. In a UK based system that would equate to a cost of between $£ 16$ to $£ 34$ per patient. 
As this is a feasibility study, it has been helpful to identify potential problems allowing us make plans to mitigate them in future studies [22].

\section{Conclusion}

In conclusion, the app would benefit from further development in terms of a more targeted and systematic incorporation of behavioural theory and techniques (such as goal setting) and individualised feedback (e.g. personal exercise goals met) to improve compliance. A broader randomised clinical trial with comparable patients to confirm clinical and wider patient lifestyle benefits of the further refined Fit 4 Surgery app is warranted.

\section{Additional file}

Additional file 1: Users guide to Fit 4 Surgery app booklet. (ZIP 1123 kb)

\section{Abbreviations}

APP: Software application; BMI: Body mass index; COPD: Chronic Obstructive Pulmonary disease; DVD: Digital versatile disc; ECOG: Eastern cooperative oncology Group performance status; ISWT: Incremental shuttle walk test; ITU: Intensive therapy unit; mMRC: Modified medical research council dyspnoea scale; NHS: National Health Service; PPC: Postoperative pulmonary complications; QOL: Quality of Life; RESTful: Representational State Transfer; ROC: Rehabilitation for operated cancer

\section{Acknowledgements}

We would like to thank Dr. Sarah Flanagan at the University of Birmingham for her advice in categorising free text responses to questionnaires and second review of these responses. We would like to thank Miss Alina-Maria Budacan MRCSEd for her contribution towards the final version of this manuscript.

\section{Authors' contributions}

SK has consented the patients in this study, collected data and contributed to writing the manuscript. BC analysed, interpreted the data and was a major contributor to the writing of the manuscript; AK consented the patients in this study, collected data and provided advice on the education element on the APP. SF conducted and analysed the qualitative interviews. CG was the lead in producing the APP and SL provided expert advice on the exercises on the app and is the lead for the pulmonary rehabilitation classes. All authors read and approved the final manuscript.

\section{Funding}

Health Foundation Grant number: CRM 1838 GIFTS 7471.

\section{Availability of data and materials}

The datasets used and/or analysed during the current study are available from the corresponding author on reasonable request.

\section{Ethics approval and consent to participate}

Ethics Committee Approval: NRES Committee West Midlands- Edgbaston REC reference 10/H1208/41. All patients included in this study gave consent to participate.

\section{Consent for publication}

Not applicable.

\section{Competing interests}

The authors declare that they have no competing interests.

\section{Author details}

${ }^{1}$ Department of Thoracic Surgery Research, Heartlands Hospital, Bordesley Green East, Birmingham B9 5SS, UK. ${ }^{2}$ Institute of Inflammation and Ageing,
College of Medical and Dental Sciences, Centre for Translational Inflammation Research, University of Birmingham Laboratories, Queen Elizabeth Hospital Birmingham, Edgbaston, Birmingham B15 2TT, UK. ${ }^{3}$ Institute of Applied Health Research, University of Birmingham, Edgbaston B15 2TT, UK. ${ }^{4}$ Evolyst- The Innovation Centre, Warwick Technology Park, Gallows Hill, Warwick CV34 6UW, UK

Received: 20 March 2019 Accepted: 24 June 2019

Published online: 05 July 2019

\section{References}

1. Lugg ST, Agostini PJ, Tikka T, Kerr A, Adams K, Bishay E, et al. Long-term impact of developing a postoperative pulmonary complication after lung surgery. Thorax. 2016 Feb;71(2):171-6.

2. West D, Woolhouse I, Beckett P, Lim, E. Y, P., Belcher E. The Thoracic Surgery Registry Brief Report Audit Years 2011-12 to 2013-14. 2015.

3. Bobbio A, Chetta A, Ampollini L, Primomo GL, Internullo E, Carbognani $P$, et al. Preoperative pulmonary rehabilitation in patients undergoing lung resection for non-small cell lung cancer. Eur J Cardiothorac Surg. 2008;33(1):95-8

4. Batchelor TJP, Rasburn NJ, Abdelnour-Berchtold E, Brunelli A, Cerfolio RJ, Gonzalez M, et al. Guidelines for enhanced recovery after lung surgery: recommendations of the enhanced recovery after surgery (ERASVR ) society and the European Society of Thoracic Surgeons (ESTS). Eur J Cardiothorac Surg. 2019;55(1):91-115.

5. Sebio Garcia R, Yáñez Brage Ml, Giménez Moolhuyzen E, Granger CL, Denehy L. Functional and postoperative outcomes after preoperative exercise training in patients with lung cancer: a systematic review and meta-analysis. Interact Cardiovasc Thorac Surg. 2016;23(3):486-97.

6. Osoba D, Rodrigues G, Myles J, Zee B, Pater J. Interpreting the significance of changes in health-related quality-of-life scores. J Clin Oncol. 1998 Jan; 16(1):139-44.

7. Bradley A, Marshall A, Stonehewer L, Reaper L, Parker K, Bevan-Smith E, et al, Pulmonary rehabilitation programme for patients undergoing curative lung cancer surgery. Eur J Cardiothorac Surg. 2013;44(4):e266-71.

8. Patel RK, Sayers AE, Patrick NL, Hughes K, Armitage J, Hunter IA. A UK perspective on smartphone use amongst doctors within the surgical profession. Ann Med Surg. 2015;4(2):107-12.

9. Ventola CL. Mobile devices and apps for health care professionals: uses and benefits. P T. 2014 May;39(5):356-64.

10. Borg G. Borg'S perceived exertion and pain scales.: Human kinetics; 1998.

11. Kelly MP, Abraham C. Behaviour change: the NICE perspective on the NICE guidance; 2009

12. Morano MT, Araújo AS, Nascimento FB, da Silva GF, Mesquita R, Pinto JS, et al. Preoperative pulmonary rehabilitation versus chest physical therapy in patients undergoing lung cancer resection: a pilot randomized controlled trial. Arch Phys Med Rehabil. 2013;94(1):53-8.

13. Jones LW, Peddle CJ, Eves ND, Haykowsky MJ, Courneya KS, Mackey JR, et al. Effects of presurgical exercise training on cardiorespiratory fitness among patients undergoing thoracic surgery for malignant lung lesions. Cancer. 2007;110(3):590-8

14. Spruit MA, Janssen PP, Willemsen SC, Hochstenbag MM, Wouters EF. Exercise capacity before and after an 8-week multidisciplinary inpatient rehabilitation program in lung cancer patients: a pilot study. Lung Cancer. 2006;52(2):257-60

15. King AC, Hekler EB, Grieco LA, Winter SJ, Sheats JL, Buman MP, et al. Harnessing different motivational frames via mobile phones to promote daily physical activity and reduce sedentary behavior in aging adults. PLoS One. 2013;8(4):e62613.

16. Vorrink SN, Kort HS, Troosters T, Lammers JW. A Mobile phone app to stimulate daily physical activity in patients with chronic obstructive pulmonary disease: development, feasibility, and pilot studies. JMIR Mhealth Uhealth. 2016 Jan 26:4(1):e11.

17. Calvo GS, Gómez-Suárez C, Soriano J, Zamora E, Gónzalez-Gamarra A, González-Béjar M, et al. A home telehealth program for patients with severe COPD: the PROMETE study. Respir Med. 2014;108(3):453-62.

18. Maddison R, Pfaeffli $L$, Stewart $R$, Kerr A, Jiang $Y$, Rawstorn J, et al. The HEART Mobile phone trial: the partial mediating effects of self-efficacy on physical activity among cardiac patients. Front Public Health. 2014; May 27;2:56. 
19. Direito A, Jiang Y, Whittaker R, Maddison R. Smartphone apps to improve fitness and increase physical activity among young people: protocol of the apps for IMproving FITness (AIMFIT) randomized controlled trial. BMC Public Health. 2015;15(1):635.

20. Harries T, Eslambolchilar P, Rettie R, Stride C, Walton S, van Woerden $H C$. Effectiveness of a smartphone app in increasing physical activity amongst male adults: a randomised controlled trial. BMC Public Health. 2016;16(1):925.

21. Chan C. LungFIT: validating a smartphone application for pulmonary rehabilitation; 2014.

22. Wisniewski SR, Leon AC, Otto MW, Trivedi MH. Prevention of missing data in clinical research studies. Biol Psychiatry. 2006;59(11):997-1000.

\section{Publisher's Note}

Springer Nature remains neutral with regard to jurisdictional claims in published maps and institutional affiliations.

Ready to submit your research? Choose BMC and benefit from:

- fast, convenient online submission

- thorough peer review by experienced researchers in your field

- rapid publication on acceptance

- support for research data, including large and complex data types

- gold Open Access which fosters wider collaboration and increased citations

- maximum visibility for your research: over $100 \mathrm{M}$ website views per year

At BMC, research is always in progress.

Learn more biomedcentral.com/submissions 